\title{
GENERALIZED LOCAL INTEGRABILITY
}

\section{ROBERT WELLAND}

1. The space of locally integrable functions (functions Lebesgue integrable on the interval $(-n, n)$ for $n=1,2, \cdots)$, as a generalization of the space of Lebesgue integrable functions contains, for instance, the polynomials but not the rational functions. The extent to which this situation can be improved by various changes in the definition of local integrability is discussed here under somewhat more general circumstances. A set $D$ of definitions of local integrability for a measure space is considered. A definition in $D$ leading to a maximal set of locally integrable functions exists if and only if the measure space is totally atomic (every measurable set is the union of atoms). When the measure space is locally compact and $\mu$ is a Radon measure [1], there exist definitions in $D$ whose resulting space of locally integrable functions contains exceptional sets of normally nonintegrable functions.

2. Let $(X, \delta, \mu)$ be a measure space. Here $X$ is a point set, $\delta$ is $\sigma$-ring of subsets of $X$ and $\mu$ is a positive measure on $\mathcal{S}$. A cover $\mathcal{C}$ of $X$ if a subset of $\delta$ such that each element in $\delta$ of finite measure is contained in some countable union of sets from $\mathfrak{C}$. To each cover $\mathfrak{C}$ of $X$, is assigned the space $\Omega_{\mathfrak{C}}$ of $\mathfrak{C}$-locally integrable functions. These are the real valued functions $f$ on $X$ such that $\chi_{E} \cdot f$ is integrable for every $E$ in $\mathcal{e}\left(\chi_{E}\right.$ is the characteristic function of $E$ ). The functions in $\Omega_{\mathfrak{C}}$ need not be measurable. For, suppose $X \notin \mathcal{S}$ and $\mathfrak{e}$ contains only sets of finite measure. $\Omega_{\mathfrak{C}}$ contains the constant functions; in this case, these are not measurable. Each cover of $X$ is regarded as a definition of local integrability; the set of such covers is denoted by $D$.

Let $\Phi_{\mathfrak{C}}$ be the space of $\mathcal{C}$-locally measurable functions; these are functions $f$ such that $f \cdot \chi_{E}$ is measurable for every $E \in \mathbb{e}$. If $X$ is a $\sigma$-finite measure space it can be shown, rather easily, that $\Phi_{\mathcal{C}}$ is independent of the cover $\mathcal{C}$ of $X$; that is, $\Phi_{\mathfrak{C}}=\Phi_{\mathfrak{C}}$, for any two covers $\mathfrak{e}$ and $\mathfrak{e}^{\prime}$.

The spaces $\Omega_{\mathbb{C}}$ are partially ordered by the inclusion relation; thus, a partial order is induced in $\mathscr{D} ; \mathfrak{C} \leqq \mathfrak{C}^{\prime}$ if $\Omega_{\mathfrak{C}} \subset \Omega_{\mathbb{C}^{\prime}}$.

Theorem 1. If $X$ is a $\sigma$-finite measure space, then $D$ contains a maximal element if and only if $X$ is totally atomic.

Proof. If $X$ is totally atomic, let $\mathfrak{C}$ be the set of atoms in $\mathcal{S}$. The

Received by the editors May 24, 1961. 
set is a cover of $X$. If $f \in \Phi_{\mathfrak{C}}$, then $f$ restricted to any element in $\mathfrak{e}$ is essentially constant. Thus, since each atom has finite measure, these restrictions are integrable. This implies $f \in \Omega_{\mathfrak{C}}$ and hence that $\Phi_{\mathfrak{C}} \subset \Omega_{\mathfrak{C}}$. Since $\Omega_{\mathfrak{C}} \subset \Phi_{\mathfrak{C}}$ we have $\Omega_{\mathfrak{C}}=\Phi_{\mathfrak{C}}$ and consequently that $\mathfrak{C}$ is a maximal element in $D$.

Suppose $X$ is not totally atomic. Let $\mathfrak{e}$ be any cover of $X$. We shall find a cover $\mathfrak{C}^{\prime}$ such that $\Omega_{\mathfrak{C}} \subset \Omega_{\mathfrak{C}}$, but $\Omega_{\mathfrak{C}} \neq \Omega_{\mathfrak{C}}$. This of course implies that $\mathcal{C}$ is not maximal and hence that $\mathbb{D}$ contains no maximal element. The assumption on $X$ ensures the existence of a least one set $E^{\prime}$ in $S$ of positive measure which contains no atoms. There exists a set $E^{\prime \prime}$ in $\mathcal{C}$ which meets $E^{\prime}$ in a set of positive measure. Let $E$ be any subset of $E^{\prime} \cap E^{\prime \prime}$ with finite positive measure.

The set $E$ contains no atoms and has positive measure; therefore, it contains a subset $F$ such that $0<\mu(F)<\mu(E)$. One of the sets $F$ or $E \sim F$ has measure less than $\mu(E) / 2$; denote it by $E_{1}$. Define $E_{n+1}$, by splitting $E_{n}$ into two subsets of positive measure and choosing the one whose measure is less than $\mu\left(E_{n}\right) / 2$. Let $\bar{E}_{0}=E \sim E_{1}$ and $\bar{E}_{n}=E_{n+1}$ $\sim E_{n}$.

If

$$
\mathfrak{C}^{\prime}=(A \cap X \sim E: A \in \mathfrak{C}) \cup\left(\bar{E}_{n}: n=0,1, \cdots\right),
$$

then $\mathfrak{e}^{\prime}$ is a cover of $X$. If $f$ is such that

$$
f(x)=1 / \mu\left(\bar{E}_{n}\right) \text { if } x \in \bar{E}_{n}, \quad n=0,1, \ldots
$$

and 0 otherwise, then $f \in \Omega_{\mathfrak{C}}$, but $f \notin \Omega_{\mathfrak{C}}$; its restriction to $E^{\prime \prime}$ is not integrable. Thus, $\Omega_{\mathfrak{C}} \neq \Omega_{\mathfrak{C}^{\prime}}$. It is easy to show that $\Omega_{\mathfrak{C}} \subset \Omega_{\mathfrak{C}^{\prime}}$, which will complete the proof.

Two elements $f, g$ in $\Omega_{\mathfrak{C}}$ are e-equivalent if $\chi_{E} \cdot(f-g)=0$ a.e. for every $E$ in $\mathcal{C}$; let $\bar{\Omega}_{\mathfrak{C}}$ be the space of $\mathcal{C}$-equivalence classes of elements in $\Omega_{\mathfrak{C}}$. If $\nu$ is another measure on $S$, let $L^{1}(X, \nu)$ be the space of equivalence classes, in the usual sense, of $\nu$-integrable functions. The next result shows that the spaces $\bar{\Omega}_{\mathcal{C}}$ and $L^{1}(X, \nu)$ are essentially always different; i.e., the study of the spaces $\Omega_{\mathfrak{C}}$ is distinct from the study of $L^{1}$ spaces.

Before proceeding to the next result, observe that to $\mathcal{C}$ can be added finite unions of sets in $\mathfrak{C}$ and subsets in $\mathcal{S}$ of sets in $\mathfrak{C}$ without changing $\Omega_{\mathfrak{C}}$. Thus, we assume $\mathcal{C}$ is closed with respect to these two properties from now on; i.e., a subset in $\mathcal{S}$ of any set in $\mathcal{C}$ is also a set in $\mathfrak{C}$ and $\mathfrak{C}$ is closed with respect to taking finite unions.

TheOREM 2. Suppose $\mathfrak{e}$ is a cover of $X$ in which there are sets $E_{1} \subset E_{2} \subset \cdots$ such that $E_{0}$ in $\mathfrak{e}$ implies $E_{0} \subset E_{n}$ for some $n$ and $\mu\left(E_{n+1} \sim E_{n}\right)>0$ for every $n$. Then $\bar{\Omega}_{\mathcal{C}} \neq L^{1}(X, \nu)$ for any measure $\nu$. 
Assume here that $\mu$ and $\nu$ are both $\sigma$-finite measures on $X$ or that $\mu$ and $\nu$ are both Radon measures and $X$ is locally compact.

Proof. Suppose there exists a measure $\nu$ such that $\bar{\Omega}_{\mathbb{C}}=L^{1}(X, \nu)$ $=L^{1}$. On the one hand, with the uniformity $\tau_{1}$ given by the norm

$$
\|f\|=\int|f| d \mu, \quad f \in L^{1},
$$

the space $L^{1}$ is a Banach space; while, on the other hand, $\bar{\Omega}_{\mathbb{C}}$ with the uniformity $\tau_{2}$ given by the semi-norms

$$
\|f\|_{n}=\int_{E_{n}}|f| d \mu, \quad f \in \bar{\Omega} \mathfrak{C},
$$

is a Fréchet space (complete metric vector space). It is an easy matter to show that the uniformity $\tau_{2}$ cannot be given by any norm [2]. It must be kept in mind that $\mu\left(E_{n+1} \sim E_{n}\right)>0$ for $n=1,2, \cdots$.

These two uniformities are compatible with the order relation; i.e., bases for the neighborhood systems at 0 exist whose elements contain with the function $f$ every function $g$ such that $|g| \leqq|f|$. It can be shown as in $[4]$ that $\left(\bar{\Omega}_{\mathbb{C}}, \tau_{2}\right)$ and $\left(L^{1}, \tau_{1}\right)$ both have the order dual as topological dual. Recall, the order dual is the space of linear forms each of which is the difference of two positive linear forms. Having the same topological dual, these two spaces have the same Mackey topology [3]. But the uniformities $\tau_{1}$ and $\tau_{2}$ are already Mackey topologies for $L^{1}$ and $\Omega_{\mathfrak{C}}$ respectively; for, with $\tau_{1}, L^{1}$ is a Banach space and with $\tau_{2}, \Omega_{\mathfrak{C}}$ is a Fréchet space [3]. Hence the uniformity $\tau_{1}$ is equivalent to $\tau_{2}$. Since $\tau_{1}$ is given by a norm, while $\tau_{2}$ cannot be given by a norm, this is a contradiction.

3. Suppose $X$ is locally compact and $\mu$ is a Radon measure. A function $f$ on $X$ is unbounded at $x$ if $\mu([x:|f(x)|>n] \cap V)>0$ for every neighborhood $V$ of $x$ and $n=1,2, \cdots$. The set of unbounded points for $f$ is denoted $\bar{U}(f)$, and the set of unbounded points for a set $F$ of functions is denoted $U(F) ; U(F)=\bigcup_{f \in F} \bar{U}(f)$.

Theorem 3. If $F \subset \Phi_{\mathfrak{C}}$ and $\mu(U(F))=0$, then there exists a cover $\mathfrak{C}$ of $X$ such that $F \subset \Omega_{\mathbb{C}}$.

Proof. Suppose $U(F)=E$ has measure zero. There exists a sequence $V_{1} \supset V_{2} \supset \cdots$ of open sets such that $E \subset V=\bigcap_{n=1}^{\infty} V_{n}$ and $\mu(V)=0$. Let $V_{n}^{\prime}$ be the complement of $V_{n}$ for $n=1,2, \cdots$. For each $n$ let $K\left(V_{n}\right)$ be the set of compact subsets of $V_{n}^{\prime}$. Let $\mathfrak{e}=\left[\cup_{n=1}^{\infty} K\left(V_{n}\right)\right] \cup[V]$. $\mathcal{e}$ is a cover of $X$; let us show $F \subset \Omega_{\mathfrak{C}}$. This 
will be done if we show that each function of $F$ when restricted to any set in $\bigcup_{n=1}^{\infty} K\left(V_{n}\right)$ is essentially bounded. Thus, let $A$ be such a set and $f$ a function in $F . A$ is compact and contains no unbounded points of $f$. Therefore, a finite set of open sets $U_{1}, U_{2}, \cdots, U_{m}$ and constants $k_{1}, k_{2}, \cdots, k_{m}$ exist such that

$$
\mu\left(\left[x:|f(x)|>d_{n}\right] \cap U_{n}\right)=0, \quad n=1,2, \cdots, m
$$

and $A \subset \mathrm{U}_{n=1}^{m} U_{n}$. This implies the restriction of $f$ to $A$ is essentially bounded.

When $X$ is a locally compact group and $\mu$ is a Radon measure, we have the stronger result

THEOREM 4. If $F \subset \Phi_{\mathcal{C}}$ and $\mu(U(F))$ has local measure zero $(\mu(U(F) \cap K)=0$ for every compact subset $K$ of $X)$, then there exists a cover $\mathfrak{e}$ of $X$ such that $F \subset \Omega_{\mathfrak{C}}$.

Proof. Suppose $U(F)$ has local measure zero. The group $X$ is the union of a pairwise disjoint set $\left(X_{\alpha}\right)_{\alpha \in A}$ of $\sigma$-compact open and closed subsets [5]. Thus, $U(F) \cap X_{\alpha}$ has measure zero for each $\alpha$ in $A$. Theorem 3 may be applied to each of the $X_{\alpha}$ to obtain a cover $\mathfrak{e}_{\alpha}$ of $X_{\alpha}$ with the property that $f$ restricted to any element of $\mathfrak{C}_{\alpha}$ is integrable for every $f$ in $F$. The set $\mathfrak{e}=\bigcup_{\alpha \in A} \mathfrak{C}_{\alpha}$ is a cover of $X$ such that $F \subset \Omega_{\mathfrak{C}}$.

In Theorem 3, the case where $\mu(U(F))>0$ is left open. Some insight into this case can be gained by considering the unit interval $I$ with Lebesgue measure. On $I$, let $L^{1}$ be the space of Lebesgue integrable functions and $\Phi$ the space of Lebesgue measurable functions. For any $a$ in $I$, let $f_{a}$ be the function whose value at $a$ is 0 and whose value at $x$ is $1 /(|x-a|)^{1 / 2}$ if $x \neq a$. For each $a$ in $I, f_{a}$ is unbounded at $a$ and $f_{a} \in L^{1}$; therefore, $\mu\left(U\left(L^{1}\right)\right)=\mu(U(\Phi))=1$. It is clear that $L^{1}=\Omega_{(I)}$; let us show that $\Phi$ is not contained in $\Omega_{\mathfrak{C}}$ for any cover $\mathcal{C}$ of $I$. This follows from two facts: first, for any set $A$ of positive measure in $I$ there exists a function $f$ in $\Phi$ such that $f \cdot \chi_{A} \notin L^{1}$ and second, any cover $\mathcal{C}$ of $I$ must contain at least one set of positive measure.

The interest in this paper is mainly in nonintegrable functions; accordingly, we say a measurable function $f$ is strictly unbounded at a point $a$ if, for every neighborhood $V$ of $a,|f| \cdot \chi_{V}$ is not integrable. Even with this stronger notion of unboundedness the converse of Theorem 3 fails.

ExAmple. There exists a countable set $F$ of finite valued Lebesgue measurable functions on $I$ and $a$ cover $\mathfrak{C}$ of $I$ such that $F \subset \Omega_{\mathfrak{C}}$, yet the set of points where the functions in $F$ are strictly unbounded has measure 1 .

Let $C$ be the subset of $I$ obtained by removing from $I$ the closed 
middle interval of length $1 / 4$, then removing the closed middle intervals of length $1 / 16$ from each of the two remaining semi-closed intervals and so on. The set $C$ clearly has measure $1 / 2$; it is to be the first element in the cover $\mathcal{C}$. The closed intervals which were removed are to be denoted by $A_{1}, A_{2}, \cdots$. Define $f$ so that $f(x)=0$ if $x \in C$, and $f(x)=1 / \mu\left(A_{j}\right)$ if $x \in A_{j}$. This function is measurable; its restriction to $C$ is integrable, and it is strictly unbounded at every point of $C$. Each of the sets $A_{j}$ is a copy of $I$ and therefore, we can apply the above process to each. A set $C_{j}$ is obtained which has measure $1 / 2 \mu\left(A_{j}\right)$. A function $f_{j}$ can be defined with support in $A_{j} \sim C_{j}$ as $f$ was defined. It will be measurable, its restriction to $C_{j}$ will be integrable and it will be strictly unbounded at each point of $A_{j}$. Furthermore, this function's restriction to $C$ is integrable for its support is contained in the complement of $C$. The restriction of $f$ to $C_{j}$ is integrable, for it is a finite constant on this set. To obtain the set $C_{j}$, we removed from $A_{j}$ a sequence of closed intervals $\left[A_{j k}\right]$. We apply to the $A_{j k}$ the same process we applied to $A_{j}$, obtaining a set $C_{j k}$ of measure $1 / 2 \mu\left(A_{j k}\right)$ and a function $f_{j k}$. Continuing in this way, we obtain, after reindexing, a pairwise disjoint sequence of sets $B_{1}$, $B_{2}, \cdots$ and a sequence of measurable functions $f_{1}, f_{2}, \cdots$ such that $\mu\left(\cup_{i=1}^{\infty} B_{i}\right)=1, f_{i} \cdot \chi_{B i}$ is integrable and $f_{i}$ is strictly unbounded at every point of $B_{i}$. Let $B_{0}=I \sim \bigcup_{i=1}^{\infty} B_{i}, \mathcal{e}=\left[B_{0}, B_{1}, B_{2}, \cdots\right]$ and $F=\left[f_{1}, f_{2}, \cdots\right]$. First, $\mathcal{e}$ is a cover of $I$, second, $F \subset \Omega_{\mathfrak{C}}$ and third, the set of strictly unbounded points of functions in $F$ has measure 1.

\section{BiBLIOGRAPHY}

1. N. Bourbaki, Intégration, Actualités Sci. Ind. No. 1175, Hermann, Paris, 1957.

2. - Espaces vectoriels topologiques, Actualités Sci. Ind. No. 1189, Hermann, Paris, 1953.

3. — Espaces vectoriels topologiques, Actualités Sci. Ind. No. 1229, Hermann. Paris, 1955, pp. 70-71.

4. C. Goffman, Compatible seminorms in a vector lattice, Proc. Nat. Acad. Sci. U.S.A. 42 (1956), 536-538.

5. L. H. Loomis, An introduction to abstract harmonic analysis, Van Nostrand, New York, 1953, p. 109.

The Ohio State University 